\title{
Characterization of genetic and phenotypic heterogeneity of obstructive sleep apnea using electronic health records
}

Olivia J. Veatch ${ }^{1,2,3^{*}}$, Christopher R. Bauer ${ }^{4}$, Brendan T. Keenan ${ }^{1}$, Navya S. Josyula ${ }^{4}$, Diego R. Mazzotti ${ }^{1}$, Kanika Bagai ${ }^{2}$, Beth A. Malow ${ }^{2}$, Janet D. Robishaw ${ }^{5}$, Allan I. Pack ${ }^{1}$ and Sarah A. Pendergrass ${ }^{4}$

\begin{abstract}
Background: Obstructive sleep apnea (OSA) is defined by frequent episodes of reduced or complete cessation of airflow during sleep and is linked to negative health outcomes. Understanding the genetic factors influencing expression of OSA may lead to new treatment strategies. Electronic health records (EHRs) can be leveraged to both validate previously reported OSA-associated genomic variation and detect novel relationships between these variants and comorbidities.

Methods: We identified candidate single nucleotide polymorphisms (SNPs) via systematic literature review of existing research. Using datasets available at Geisinger $(n=39,407)$ and Vanderbilt University Medical Center $(n=24$, 084), we evaluated associations between 40 previously implicated SNPs and OSA diagnosis, defined using clinical codes. We also evaluated associations between these SNPs and OSA severity measures obtained from sleep reports at Geisinger $(n=6571)$. Finally, we used a phenome-wide association study approach to help reveal pleiotropic genetic effects between OSA candidate SNPs and other clinical codes and laboratory values available in the EHR.

Results: Most previously reported OSA candidate SNPS showed minimal to no evidence for associations with OSA diagnosis or severity in the EHR-derived datasets. Three SNPs in LEPR, MMP-9, and GABBR1 validated for an association with OSA diagnosis in European Americans; the SNP in GABBR1 was associated following meta-analysis of results from both clinical populations. The GABBR1 and LEPR SNPs, and one additional SNP, were associated with OSA severity measures in European Americans from Geisinger. Three additional candidate OSA SNPs were not associated with OSA-related traits but instead with hyperlipidemia and autoimmune diseases of the thyroid.

(Continued on next page)
\end{abstract}

\footnotetext{
* Correspondence: oveatch@kumc.edu

'Division of Sleep Medicine/Department of Medicine, Perelman School of

Medicine at the University of Pennsylvania, 125 S. 31st St, Office 2123,

Philadelphia, PA 19104, USA

${ }^{2}$ Sleep Disorders Division/Department of Neurology, Vanderbilt University

Medical Center, Nashville, TN 37232, USA

Full list of author information is available at the end of the article
}

(c) The Author(s). 2020 Open Access This article is licensed under a Creative Commons Attribution 4.0 International License, which permits use, sharing, adaptation, distribution and reproduction in any medium or format, as long as you give appropriate credit to the original author(s) and the source, provide a link to the Creative Commons licence, and indicate if changes were made. The images or other third party material in this article are included in the article's Creative Commons licence, unless indicated otherwise in a credit line to the material. If material is not included in the article's Creative Commons licence and your intended use is not permitted by statutory regulation or exceeds the permitted use, you will need to obtain permission directly from the copyright holder. To view a copy of this licence, visit http://creativecommons.org/licenses/by/4.0/ The Creative Commons Public Domain Dedication waiver (http://creativecommons.org/publicdomain/zero/1.0/) applies to the data made available in this article, unless otherwise stated in a credit line to the data. 


\begin{abstract}
(Continued from previous page)
Conclusions: To our knowledge, this is one of the largest candidate gene studies and one of the first phenomewide association studies of OSA genomic variation. Results validate genetic associates with OSA in the LEPR, MMP-9 and GABBR1 genes, but suggest that the majority of previously identified genetic associations with OSA may be false positives. Phenome-wide analyses provide evidence of mediated pleiotropy. Future well-powered genomewide association analyses of OSA risk and severity across populations with diverse ancestral backgrounds are needed. The comprehensive nature of the analyses represents a platform for informing future work focused on understanding how genetic data can be useful to informing treatment of OSA and related comorbidities.
\end{abstract}

Keywords: Sleep disorders, Obstructive sleep apnea, Genetics, Pleiotropy, Electronic health records

\section{Background}

Obstructive sleep apnea (OSA) is defined by frequent episodes of reduced (hypopnea) or complete (apnea) cessation of airflow that occur due to upper airway obstruction during sleep, and is among the most common sleep disorders in the world [1]. When left untreated, OSA represents a significant public health burden, carrying a higher risk of serious comorbidities such as cardiovascular disease [2], cancer [3], cognitive impairment [4], and rate of progression of neurodegeneration [5]. As such, identifying more effective diagnosis and management of OSA are important research areas.

Understanding the genetic mechanisms contributing to expression of OSA and related comorbidities offers the opportunity to inform novel, personalized treatment approaches. Studies indicate that OSA is heritable [6], providing evidence for genetic influences. Genomic variation is also implicated in wellestablished structural risk factors for OSA (e.g., soft tissue volumes [7], craniofacial dimensions [8], and obesity [9]). Furthermore, numerous syndromes with well-defined genetic causes are associated with increased prevalence of OSA (e.g., Achondroplasia [10], Down syndrome [11], Marfan's syndrome [12], and Prader-Willi syndrome [13]). Despite the evidence implicating genetic factors in OSA, no strong candidates are established. This is possibly due to underpowered studies, lack of replication, and the wide variability of OSA symptomatology and comorbidities within evaluated patient populations.

With the expansion of biorepositories linked to electronic health records (EHRs), large sample sizes and a variety of OSA-related information-as well as other clinical data-are available, offering unprecedented opportunity to establish more robust genetic associations. In addition, phenome-wide association studies (PheWAS) can help reveal pleiotropic genetic effects influencing expression of OSA and/or co-occurring conditions by testing variants for associations with a broad range of EHR-derived phenotypes [14]. In addition to uncovering pleiotropy, identifying candidate variants associated with OSA-related comorbidities, but not OSA itself, may suggest the original associations were due to unrecognized confounding.

Therefore, in the present study we used EHRs linked to genomic data to determine if previously reported associations between single nucleotide polymorphisms (SNPs) and OSA validated in clinical samples in the United States. We then used PheWAS to provide evidence of pleiotropic effects for OSA-associated SNPs, or to determine whether previous evidence of associations with OSA may actually reflect relationships with underlying comorbidities. This work represents one of the largest candidate studies, and the first PheWAS, focused on OSA genetics.

\section{Methods}

Search strategy and selection criteria for OSA-associated genomic variants

To identify OSA candidate genetic variants, PubMed was queried for original research papers and previous reviews (up to January 1, 2018) that focused on genetic risk factors for OSA. Search terms for PubMed queries were as follows: (obstructive sleep apnoea [Title/Abstract] OR obstructive sleep apnea [Title/Abstract])) AND (polymorphism OR genetic variant OR genetic association OR gene OR genome wide association study OR genome-wide association study). PubMed results were filtered to include studies conducted in humans. To further expand the query space, we additionally searched the embase resource (https://www.embase. $\mathrm{com} /$ ) focusing on articles that were available via embase or MEDLINE. Search terms for embase queries required use of 'sleep disordered breathing' for disease focus, as opposed to allowing queries for 'obstructive sleep apnea', and were as follows: 'sleep disordered breathing'/mj AND ('genetic polymorphism'/mj OR 'single nucleotide polymorphism'/mj OR 'genetic association study'/mj OR 'genetic association'/mj) AND ([embase]/lim OR [medline]/lim) AND [<1966-2018]/py AND [humans]/lim AND [abstracts]/lim AND [english]/lim AND ([article]/ $\lim$ OR [letter]/lim OR [review]/lim) AND ([young adult]/lim OR [adult]/lim OR [middle aged]/lim OR [aged]/lim). Abstracts were manually reviewed to ensure 
that results related to genetic studies that were specifically relevant to OSA, as opposed to phenotypes that may reflect something other than an OSA diagnosis (e.g., treatment response). Studies were excluded if there were no full-texts available or they represented analyses of genomic regions for which individual variants could not be identified. The remaining full manuscripts were manually reviewed to identify candidate variants. Candidate variants were then mapped to reference SNP IDs (rsIDs) and the final list of candidate SNPs was pruned for linkage disequilibrium (based on $r^{2}<0.50$ ) separately in each independent dataset to identify SNPs tagging independent genomic regions. When necessary, proxy SNPs for candidate variants were identified using data from 1000 Genomes, phase III [15] Europeans, since this ancestral group represented the largest proportion of our analysis datasets using rAggr (http://raggr.usc.edu/) and an $\mathrm{r}^{2}>0.50$.

\section{Study populations}

Geisinger samples were selected from the current MyCode biobank participants. MyCode is a major resource for research that combines information obtained from DNA and serum with health information from the electronic health record (Epic) and other sources intended to improve the prevention, diagnosis, and treatment of disease [16]. No specific clinics or practices are targeted by MyCode and there is a high consent rate, suggesting samples are representative of the overall health system [16]. EHR-defined cases (for definition, see below) were required to have at least 1 year of activity post their first OSA diagnosis and be between 18 and 88 years of age at time of the OSA diagnosis. EHRdefined non-cases (for definition, see below) were required to have at least 2 years of activity in the health system between January 1, 2008 and December 31, 2016 and be between 18 and 88 years of age as of December 31, 2016.

Vanderbilt University Medical Center (VUMC) samples were selected using data from individuals in Vanderbilt's biorepository linked to electronic health records (BioVU). BioVU is a biorepository of DNA extracted from discarded blood collected during routine clinical testing and linked to de-identified medical records in the Synthetic Derivative. As BioVU samples are obtained from every clinic that collects blood for routine laboratory tests at VUMC, we expect minimal bias with regard to the clinical aspects of these samples [17]. The Synthetic Derivative is a de-identified copy of the main hospital electronic health record databases created for research purposes. De-identification was achieved primarily through the application of a commercial electronic program, which was applied and assessed for acceptable effectiveness in scrubbing identifiers.
Individuals included in the study were required to have available genome-wide genotyping data and at least 2 years of activity in the health system between January 1, 2001 and June 6, 2017. EHR-defined cases were additionally required to have be between 18 and 88 years of age at time of the first code usage. EHR-defined noncases were required to be between 18 and 88 years of age at the time of data analysis.

Analyses were performed across three independent datasets from the two sites (i.e., Geisinger European Americans [Geisinger] and Vanderbilt University Medical Center European Americans [VUMC-EA] and African Americans [VUMC-AA]). The Geisinger dataset was comprised of 39,407 individuals with European ancestry, and the VUMC datasets included 20,688 individuals with European ancestry and 3396 individuals with African ancestry (Table 1). Other ancestral groups were not included due to limited sample sizes at Geisinger and VUMC.

\section{Genotype quality control and imputation}

Genotype data for the Geisinger dataset were generated using the Illumina HumanOmniExpressExome bead chip. Genotype data for the VUMC datasets were generated using the Illumina ${ }^{\circ}$ Multi-Ethnic Global Array (MEGA). Quality control (QC) procedures required DNA samples to have $>90 \%$ genotyping call rate and be unrelated based on PI-HAT $\leq 0.05$. Directly genotyped markers were required to have $>99 \%$ call rate with minor allele frequency $>0.01$, and $p$-values testing significant deviation from Hardy-Weinberg Equilibrium > $1.0 \times 10^{-7}$. Genetically-informed ancestry was determined using principal components analysis with reference human genomes available via the 1000 Genomes Project, phase III [15]. The quality controlled data for the European dataset from Geisinger were imputed using Impute 2 and reference human haplotypes available via the Haplotype Reference Consortium [18]. The quality controlled data for the European and African VUMC datasets were imputed using reference human haplotypes available via the 1000 Genomes Project, phase III [15]. Imputed genotypes were required to have info scores $\geq 0.3$. Notably, while all other QC thresholds were required for inclusion in association tests, two imputed SNPs were missing in $>10 \%$ of the VUMC-AA dataset: rs999944 (missing $=19.3 \%$ ) and rs7804372 (missing = 10.5\%). These SNPs were not tested for associations in this dataset.

\section{EHR-Derived OSA phenotypes}

Our primary OSA phenotype was cases and non-cases status derived using an EHR algorithm based on the total instances (e.g., on different dates) of OSA-related International Classification of Diseases (ICD) 9th or 
Table 1 Comparison of Demographics across Datasets and between Cases and Controls

\begin{tabular}{|c|c|c|c|c|c|}
\hline Analysis Sample & Demographics & Totals* & $\begin{array}{l}\text { EHR-defined } \\
\text { OSA }\end{array}$ & EHR-defined No OSA & $p$-value \\
\hline \multirow{4}{*}{$\begin{array}{l}\text { Geisinger } \\
(E A)\end{array}$} & N & 39,407 & 5760 & 33,647 & - \\
\hline & $\mathrm{Age}^{\mathrm{a}}$, years & $59.3(17.3)$ & $61.1(12.6)$ & $58.9(17.2)$ & $2.65 \times 10^{-29}$ \\
\hline & $\mathrm{BMI}^{\mathrm{a}}, \mathrm{kg} / \mathrm{m}^{2}$ & $31.6(18.7)$ & $40.1(9.8)$ & $30.1(6.8)$ & $<1.58 \times 10^{-322}$ \\
\hline & Male, \% & $40.9 \%$ & $52.9 \%$ & $38.9 \%$ & $2.15 \times 10^{-87}$ \\
\hline \multirow{4}{*}{$\begin{array}{l}\text { VUMC } \\
\text { (EA) }\end{array}$} & $\mathrm{N}$ & 20,688 & 2831 & 17,857 & - \\
\hline & $\mathrm{Age}^{\mathrm{a}}$, years & $56.6(17.5)$ & $55.8(13.0)$ & $56.8(18.2)$ & $2.88 \times 10^{-4}$ \\
\hline & $\mathrm{BMI}^{\mathrm{a}}, \mathrm{kg} / \mathrm{m}^{2}$ & $28.8(7.3)$ & $34.3(9.0)$ & $27.9(6.6)$ & $1.08 \times 10^{-241}$ \\
\hline & Male, \% & $42.4 \%$ & $56.9 \%$ & $40.1 \%$ & $1.93 \times 10^{-62}$ \\
\hline \multirow{4}{*}{$\begin{array}{l}\text { VUMC } \\
\text { (AA) }\end{array}$} & N & 3396 & 331 & 3065 & - \\
\hline & $\mathrm{Age}^{\mathrm{a}}$, years & $47.5(17.3)$ & $49.2(13.8)$ & $47.3(17.6)$ & $5.73 \times 10^{-2}$ \\
\hline & $\mathrm{BMI}^{\mathrm{a}}, \mathrm{kg} / \mathrm{m}^{2}$ & $31.0(8.6)$ & $38.5(10.4)$ & $30.2(8.0)$ & $2.91 \times 10^{-36}$ \\
\hline & Male, \% & $37.8 \%$ & $41.7 \%$ & $37.4 \%$ & $1.37 \times 10^{-1}$ \\
\hline
\end{tabular}

${ }^{a}$ Age and body mass index (BMI) are presented as means (standard deviations), and for cases with EHR-defined OSA were reported in the same year as the first use of the OSA-related code, while for controls the most recent measurements were used. *Traits compared between analysis datasets were all significantly different at $p \leq 3.37 \times 10^{-7}$. EHR Electronic health record, VUMC Vanderbilt University Medical Center, AA African or EA European (North) American

10th revision codes (Table S1) in the individual's health record [19]. This algorithm was recently validated with clinical chart reviews across multiple sites in the U.S., including Geisinger and VUMC [19]. For the present study, the minimum number of code instances that achieved positive predictive value (PPV) and negative predictive value (NPV) of at least $90 \%$ was used for determining OSA cases and non-cases. At Geisinger, a case-definition of OSA-related codes on at least 3 different dates in the EHR was required to achieve PPV and $\mathrm{NPV} \geq 90 \%$ (PPV [95\% CI] =94.9 [88.5, 98.3]; NPV [95\% $\mathrm{CI}]=95.0[88.7,98.4])[19]$. At VUMC, a case-definition of OSA-related codes on at least 2 different dates achieved these thresholds (PPV $=97.5$ [92.9, 99.5]; $\mathrm{NPV}=94.0[87.4,97.8])[19]$. Non-cases were defined as zero OSA-related diagnostic codes in the EHR at both sites. Individuals not meeting the case or non-case definitions were excluded.

In addition to case and non-case status, secondary analyses were performed using 13 available phenotypes from sleep study (polysomnography [PSG]) reports available for a subset of the dataset from Geisinger $(n=4957$ EHRdefined cases and $n=1614$ non-cases); these data were not available at VUMC due to issues with deidentification of polysomnography results that impeded inclusion of these data in the Synthetic Derivative. Only data from full-night, diagnostic in-laboratory sleep studies (e.g., not split-night studies) with at least $120 \mathrm{~min}$ of total sleep time were included. If an individual had more than 1 sleep report, the diagnostic sleep study that was conducted on the date closest to the first usage of an OSA code (among EHR-defined cases) or the most recent study (among EHR-defined non-cases) was chosen. Standard American Academy of Sleep Medicine criteria was used to define hypopneas. Phenotypes included respiratory indices (Apnea/Hypopnea Index [AHI], Central Apnea Index [CAI], Obstructive Apnea Index [OAI]), mean event durations (Respiratory Event [RDI] Duration and Duration of Apnea/Hypopnea), hypoxia measures (Percent Time with $\mathrm{SaO}_{2}<89 \%$ during Non-Rapid Eye Movement [NREM] or Rapid Eye Movement [REM], Minimum $\mathrm{SpO}_{2}$ during Respiratory Event or Total Sleep Time), measures of sleep quality (Number of Awakenings, Sleep Efficiency, Wake After Sleep Onset), and subjective sleepiness (the Epworth Sleepiness Scale [ESS] [20]).

\section{EHR-derived body mass index}

Body mass index (BMI) was calculated from the EHR data at each site based on available measures of height and weight. The primary BMI value of interest was the BMI closest to the time of the first OSA code usage (in EHR-defined cases) or the most recent value (for EHRdefined controls). Site-specific quality control and data cleaning procedures were applied to assure high-quality BMI measurements.

At Geisinger, height was recorded in inches and weight in pounds; there was no evidence of outliers explained by incorrect units. Calculated BMI values $>3$ standard deviations above or below a patient's median BMI value were excluded as outliers. If fewer than three BMI measurements remained after these exclusions, all BMI data were considered unreliable and excluded. At VUMC, height and weight values were previously cleaned and obvious outliers removed based on a validated algorithm [21]. Median BMI values within the same year as the first OSA code usage (for EHR-defined cases) or most recent year of EHR data (for EHR-defined non-cases) were calculated, manually reviewed, and 
excluded as outliers if $>3$ standard deviations above or below a patient's overall median BMI.

\section{Statistical analyses}

Unless otherwise specified, continuous data are summarized as means and standard deviations and categorical data as frequencies and percentages. Age and BMI were compared between EHR-derived cases and non-cases in each dataset using $\mathrm{T}$-tests and across the Geisinger, VUMC-EA and VUMC-AA datasets using analysis of variance and Tukey-Kramer post-hoc pairwise comparisons. Sex was compared using chi-squared tests. All models were evaluated adjusting for age and BMI at the time of first OSA code usage (for EHR-defined cases) or most recently measured (for EHR-defined non-cases), as well as sex (except in sex-stratified analyses). Given the higher genetic diversity of the VUMC sample, all analyses were additionally adjusted for global ancestry using the first three principal components calculated for each dataset, as was done previously for VUMC datasets [22]. Principal component adjustment was not included in analyses of the Geisinger dataset, given the high genetic homogeneity of the sample and the fact that previous studies have not observed a difference in results when adjusting for ancestry PCs compared to unadjusted for PCs in this dataset [16].

\section{Genetic associations with OSA-related phenotypes}

Analyses were performed to evaluate the associations between candidate SNPs identified from the systematic literature review and EHR-derived OSA phenotypes. SNPs were coded additively with respect to the minor allele for the population being assessed. To maintain consistency throughout, all results are reported based on mapping to the positive strand. Primary analyses evaluated associations between candidate SNPs and EHRbased OSA diagnosis using logistic regression models, separately within each dataset. In addition to independent analyses within each sample, we performed two separate meta-analyses of the two European American datasets alone and of all three analysis samples (Geisinger, VUMC-EA, and VUMC-AA). Inverse-variance, standard error based meta-analyses, and the possibility of heterogeneity among the studies, was tested in the METAL software package (version: 2011-03-25) [23]. Given a priori evidence of associations between candidate SNPs and OSA, significant evidence of validation was set at an uncorrected $p<5.0 \times 10^{-2}$ when the initial study reporting the association with OSA was conducted in an ancestral population of comparable ancestry to our study populations. Significant evidence of generalizability was determined using the Benjamini-Hochberg (BH) method, and was based on a false discovery rate of $5 \%$ (e.g., $\mathrm{q}<5.0 \times 10^{-2}$ ) when the initial study reporting the association with OSA was conducted in an ancestral population of different ancestry than our study populations. To determine if age, sex or BMI modified the associations between any candidate SNP and EHR-derived OSA diagnosis, we performed interaction tests by including a product term (SNP $\mathrm{x}$ [covariate]) in a logistic regression model that also included both main effects (SNP, covariate). To control for the numerous interactions tested in these analyses, statistical significance was determined using the $\mathrm{BH}$ method and was set at $\mathrm{q}<$ $5.0 \times 10^{-2}$.

Secondary analyses of genetic associations with 13 OSA phenotypes from sleep study reports in the Geisinger European American sample were performed using linear regression. Given the quantitative nature of these traits and the comparatively smaller analysis sample, sleep traits were analyzed in all patients with available data regardless of EHR-defined case/control status. As the majority of these quantitative traits did not meet normality assumptions, Box-Cox power transformations were applied and then Z-scores were calculated prior to analyses. For variables that included values of 0 , half of the minimum non-zero value was added to each observation prior to transformation. Given a priori evidence of associations between candidate SNPs and OSA, we determined significance for results separately for SNPs where the initial study reporting the association was conducted in an ancestral population of comparable ancestry to our study populations versus a different ancestry. Specifically, when the initial study was conducted in either Europeans or African Americans, $p$-values from association tests between each SNP and all traits were corrected using $\mathrm{BH}$ and significant evidence of validation was set at $\mathrm{q}<5.0 \times 10^{-2}$. When the initial study was conducted in non-comparable ancestral populations, significant evidence of generalizability was determined based on correction of $p$-values from association tests between all of these SNPs and all phenotypes.

\section{Phenome-wide association study methods for OSA candidate SNPs: discovery (Geisinger) and replication (VUMC)}

To determine whether there was evidence of pleiotropic genetic effects of OSA candidate variants with other phenotypes in the EHR or whether initial associations between SNPs and OSA may be driven by underlying associations with co-occurring conditions (e.g., unmeasured confounding), we performed a phenome-wide association study (PheWAS).

Given that the PheWAS were novel analyses, as opposed to the validation studies that were conducted for previously reported OSA candidate SNPs, we chose to perform a discovery analysis in the Geisinger dataset and replication in the VUMC-EA dataset. We also tested for 
generalization to the VUMC-AA dataset. For discovery PheWAS analyses in the Geisinger sample, associations between all OSA candidate SNPs and a total of 574 nonOSA ICD codes and 143 Logical Observation Identifier Names and Codes (LOINC) mapped median laboratory values were assessed using logistic and linear regression models, respectively. For non-OSA ICD codes, only codes resulting in $\geq 200$ cases in the Geisinger dataset were included (Table S2). For each ICD code, 'cases' were defined as individuals with codes used on $\geq 3$ different dates and 'controls' were defined as individuals with absence of this code or a code within the same hierarchy. Individuals with one or two instances of a code were excluded from the analysis for that code. Box-Cox power transformations were applied to quantitative median laboratory values prior to analysis. Only laboratory values available for $\geq 1000$ individuals [24] in the Geisinger dataset were included (Table S3). Statistical significance in these discovery analyses was determined using the BH method and was set at $\mathrm{q}<5.0 \times 10^{-2}$.

Significant SNP-phenotype associations discovered in the Geisinger sample were replicated in the VUMC datasets using codes that reflected the same phenotype code (PheCode Map 1.2 [25]) as the discovery ICD code, present on $\geq 3$ different dates in the individual's record. PheCodes were used for replication analyses to circumvent issues related to potential differences in specific ICD code usage across the two clinical sites, as they represent distinct health care systems [25]. Prior to PheCode mapping, all ICD-10 codes were mapped to ICD-9 codes using CMS General Equivalency Mapping via the Agency for Healthcare Research and Quality MapIT Tool, Application Version 5.1.110, data version 2.2018.110X (https://www.qualityindicators.ahrq.gov/resources/Toolkits.aspx). Given the goal of replicating specific SNP-phenotype associations discovered in the Geisinger sample, the significance threshold for these analyses was set at $p<5.0 \times 10^{-2}$. All PheWAS tests were conducted while adjusting for age, sex and BMI as described above. In addition, analyses conducted in the VUMC datasets were conducted while also adjusting for ancestry PCs.

\section{Results}

\section{Literature-derived identification of OSA candidate variants}

The initial queries of PubMed identified 428 studies meeting the search criteria, and 221 of these met inclusion criteria after manual reviews of abstracts. Forty articles were also identified by focusing only on studies sourced from embase and MEDLINE; however, crossreferencing search results indicated that only one of these was not also included in PubMed and captured in the initial queries. Ultimately, a systematic review of 205 studies revealed 51 unique OSA candidate SNPs (Fig. S1 and Table S4). Five SNPs were dropped due to low allele frequencies $(\mathrm{MAF}<0.01)$ and six SNPs were dropped due to missingness. Analyses described below evaluated associations with 40 SNPs: 38 directly measured in our samples, and two proxy SNPs for literature-derived SNPs rs35424364 (proxy: rs7752028, $\mathrm{r}^{2}=0.97$ ) and $\mathrm{rs} 25531$ (proxy: rs11080123, $\mathrm{r}^{2}=0.50$ ).

\section{Sample characteristics}

Demographic characteristics of the analysis datasets, overall and stratified by EHR-derived OSA status, are presented in Table 1. Among the Geisinger sample, the mean (SD) age was 59.3 (17.3) years and the mean (SD) BMI was $31.6(18.7) \mathrm{kg} / \mathrm{m}^{2}$. A total of $9.5 \%$ of the Geisinger sample met Center for Disease Control criteria for severe obesity $\left(B M I \geq 40 \mathrm{~kg} / \mathrm{m}^{2}\right), 40.9 \%$ were male and $14.6 \%$ met EHR-defined criteria for OSA. Individuals with EHR-defined OSA were older, had higher BMIs and were more likely to be male than those with no evidence of OSA (Table 1). Among the VUMC-EA sample, the mean (SD) age was 56.6 (17.5) years and the mean (SD) BMI was $28.8(7.3) \mathrm{kg} / \mathrm{m}^{2} ; 7.5 \%$ of the dataset met criteria for severe obesity, and $42.4 \%$ were male. In total, $13.7 \%$ of individuals met EHR criteria for OSA. These individuals had higher BMI and were more likely to be males, but were 1 year younger on average than those with no evidence of OSA. Among the VUMC-AA sample, the mean (SD) age was 47.5 (17.3) years and the mean (SD) BMI was $31.0(8.6) \mathrm{kg} / \mathrm{m}^{2}, 13.8 \%$ met criteria for severe obesity, $37.8 \%$ were male and $9.7 \%$ met EHRdefined criteria for OSA. Individuals with EHR-defined OSA had significantly higher BMI than non-cases, but there were no significant differences in age or gender.

As shown in Table 1, participants in the Geisinger dataset had higher BMIs than both VUMC datasets. The VUMC-EA dataset had the lowest average BMI and the highest proportion of males, while the VUMC-AA dataset was the youngest and had the lowest proportion of males.

\section{Associations of OSA candidate variants with EHR-derived OSA diagnosis}

Associations between candidate SNPs and EHR-derived OSA case and non-case status were evaluated in each dataset. Of the 40 SNPs tested, SNPs in the LEPR gene (rs1137101; $p=3.78 \times 10^{-2}$ ) and $\sim 2 \mathrm{~kb}$ upstream of the $M M P-9$ gene $\left(\mathrm{rs} 3918242 ; p=2.65 \times 10^{-2}\right)$ validated in the Geisinger sample and a SNP in the GABBR1 gene (rs29230; $p=3.65 \times 10^{-2}$ ) validated in the VUMC-EA sample (Fig. 1 and Table 2). The variant in GABBR1, but not variants in $L E P R$ and $M M P-9$, was also significantly associated with EHR-derived OSA status in the metaanalysis of both European American datasets $(p=8.44 \times$ 


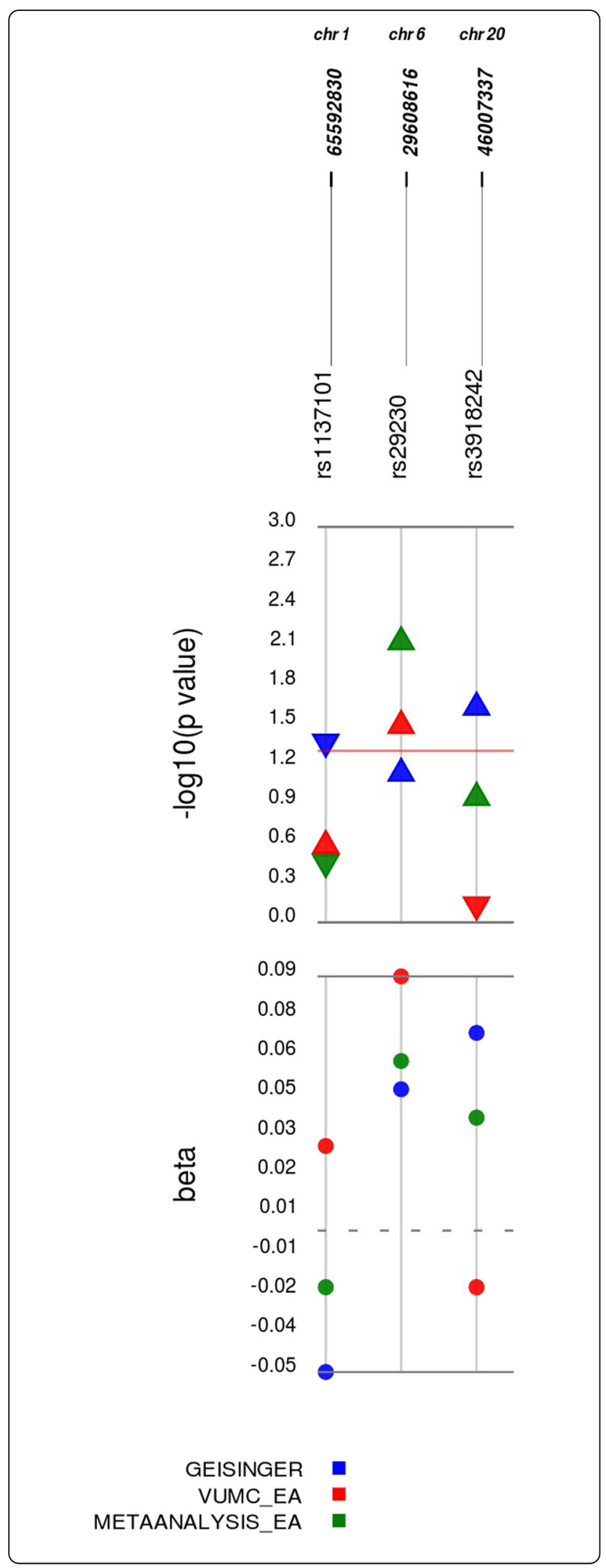

Fig. 1 Validated Associations with Obstructive Sleep Apnea Diagnosis in European Americans. Plotted are the estimates of the additive effects (beta) of the minor allele at each candidate SNP associated with a validated definition of OSA obtained from the EHR, along with the corresponding $-\log _{10} p$-value. For each SNP, the rsID, chromosome and hg38 base pair location are provided. Results from analyses conducted in the Geisinger European American (EA) dataset are plotted in blue, VUMC European Americans in red, and meta-analyses of results from tests conducted in both European American datasets in green. For $p$-values, up arrows denote increased risk for OSA diagnosis given the minor allele at this SNP and down arrows denote reduced risk. Red line denotes unadjusted significance threshold $\left(p<5.0 \times 10^{-2}\right)$

$\left.10^{-3}\right)$. No previously reported candidate SNPs validated in the EHR-derived African American dataset from VUMC (Table S4).

In addition to assessing overall associations between candidate SNPs and EHR-derived OSA diagnosis, we evaluated whether there was evidence that associations were modified by age, sex or BMI using statistical interaction tests. These analyses suggested that associations between 15 SNPs and OSA diagnosis may be modified by age, sex or BMI (Table S5). However, none of the strata-specific associations were significant after correction for multiple comparisons.

\section{Associations of OSA candidate variants with quantitative sleep traits}

In addition to associations with EHR-derived OSA diagnosis across samples, we examined relationships between candidate SNPs and quantitative OSA phenotypes available in a subset of the European American sample at Geisinger (Table S6). Three SNPs were significantly associated, following multiple testing corrections, with quantitative measures of OSA severity obtained from sleep study reports at Geisinger (Fig. 2). Of particular interest, the GABBR1 and LEPR SNPs associated with EHR-derived OSA diagnosis (Table 2) were both associated with quantitative OSA phenotypes. The GABBR1 SNP (rs29230) associated with increased risk for EHR-defined OSA diagnosis in meta-analysis was associated with increased wake after sleep onset. Additionally, the LEPR gene variant (rs1137101) associated with reduced risk for OSA diagnosis was associated with a decreased number of awakenings and wake after sleep onset, and an increased sleep efficiency (Table 2). One additional SNP, which was not associated with EHR-defined OSA diagnosis, was associated with sleep study report variables. This SNP, a non-coding variant in PTGER3 (rs1409986) previously related to increased risk for OSA, was associated with fewer awakenings, less percent time with $\mathrm{SaO}_{2}<89 \%$ in REM and decreased wake after sleep onset. 
Table 2 Validated Associations with Obstructive Sleep Apnea Diagnosis and Severity in European Americans

\begin{tabular}{|c|c|c|c|c|c|c|c|c|c|c|}
\hline \multirow[t]{2}{*}{ SNP } & \multirow[t]{2}{*}{ Chr } & \multirow{2}{*}{$\begin{array}{l}\text { Closest } \\
\text { Gene }\end{array}$} & \multirow[t]{2}{*}{ EHR-Derived Trait } & \multirow{2}{*}{$\begin{array}{l}\text { Effect } \\
\text { Allele }\end{array}$} & \multicolumn{2}{|l|}{ Geisinger } & \multicolumn{2}{|l|}{ VUMC } & \multicolumn{2}{|l|}{ Meta-Analysis } \\
\hline & & & & & $\beta$ (SE) & $p$-valuet & $\beta$ (SE) & $p$-valuet & $\beta$ (SE) & $p$-valuet \\
\hline \multirow[t]{4}{*}{ rs1137101 } & $1 \mathrm{p} 31.3$ & $\angle E P R$ & OSA Diagnosis & G & $-0.05(0.02)$ & $3.78 \times 10^{-2}$ & $0.03(0.03)$ & $2.96 \times 10^{-1}$ & $-0.02(0.02) \ddagger$ & $3.09 \times 10^{-1}$ \\
\hline & & & WASO (mins) & & $-0.07(0.02)$ & $2.64 \times 10^{-4}$ & & & & \\
\hline & & & Sleep Efficiency (\%) & & $0.06(0.02)$ & $2.28 \times 10^{-3}$ & & & & \\
\hline & & & Awakenings (\#) & & $-0.05(0.02)$ & $9.40 \times 10^{-3}$ & & & & \\
\hline \multirow[t]{2}{*}{ rs29230 } & $6 p 22.1$ & GABBR1 & OSA Diagnosis & G & $0.05(0.03)$ & $8.42 \times 10^{-2}$ & $0.09(0.04)$ & $3.65 \times 10^{-2}$ & $0.06(0.02)$ & $8.44 \times 10^{-3}$ \\
\hline & & & WASO (mins) & & $0.08(0.02)$ & $1.72 \times 10^{-3}$ & & & & \\
\hline rs3918242 & $20 q 13.12$ & MMP-9 & OSA Diagnosis & $\mathrm{T}$ & $0.07(0.03)$ & $2.65 \times 10^{-2}$ & $-0.02(0.04)$ & $6.48 \times 10^{-1}$ & $0.04(0.03)$ & $1.28 \times 10^{-1}$ \\
\hline
\end{tabular}

Shown are results for literature-derived SNPs where OSA associations reported in populations of European ancestry validated for an association with electronic health record-derived OSA-related traits in European Americans. $+p$-values are unadjusted. $\neq$ Indicates evidence for significant heterogeneity $\left(p<5.0 \times 10^{-2}\right)$. Abbreviations: SNP Single nucleotide polymorphism, Chr Chromosome, EHR Electronic health record, SE Standard error, OSA Obstructive Sleep Apnea, Resp Respiratory, WASO Wake After Sleep Onset, Mins Minutes. All SNPs evaluated in EHR-based datasets are mapped to the positive strand. More details and FDRadjusted q-values for OSA diagnosis association tests are available in Table S4 and sleep study report variables tests in Table S6

\section{Phenome-wide association studies of OSA candidate variants}

In addition to analyses of the relationship between candidate OSA SNPs and OSA-related phenotypes, to assess for possible pleiotropy or whether previous relationships with OSA may be driven by underlying associations with OSA-related comorbidities, we performed a PheWAS for each candidate SNP. None of the three SNPs that validated for an association with OSA diagnosis were associated with other non-OSA clinical traits once we controlled for multiple testing (Table S7). However, seven other candidate SNPs were associated with nonOSA clinical traits at an FDR $<5 \%$ in discovery analyses at Geisinger (Table S7). Of these, associations between three SNPs and non-OSA clinical traits replicated in the VUMC-EA dataset (Table 3). In particular, rs7412, the marker of the $A P O E-\varepsilon 2$ allele, was associated with lower risk of hyperlipidemia in both the Geisinger and VUMCEA samples. Moreover, rs429358, the marker of the $A P O E-\varepsilon 4$ allele, was associated with increased risk of hyperlipidemia in all of the EHR-derived datasets. These associations validate previously reported relationships between $A P O E$ SNPs and hyperlipidemia [26]. Finally, a variant in the HLA-DQA1 gene (rs2187668) was associated with increased risk for celiac disease and autoimmune diseases of the thyroid in both European American datasets. The association between rs2187668 and EHR-derived codes for celiac disease validates results from previous studies [27].

\section{Discussion}

By harnessing the rich resources available in electronic health records linked to genetic data, we performed a comprehensive study of the relationship between genetic variants previously implicated in OSA risk and both EHR-defined OSA status and other phenotypes derived from data in the medical record. A majority of the candidate SNPs evaluated did not show significant associations with EHR-derived OSA status in our samples, suggesting these original results could be false positive associations. However, three of the candidate variants validated among the European American samples, including variants within or near the $L E P R, M M P-9$ and $G A B B R 1$ genes. In particular, variants in $G A B B R 1$ and $L E P R$ were associated with both OSA diagnosis and severity in the EHR-derived European American datasets evaluated and may be particularly likely to translate to clinical populations of European ancestry. Ultimately, results reported here represent a first step towards leveraging EHR-based phenotypes to tease apart the heterogeneity underlying expression of OSA. We expect this may help provide the basis for more personalized treatment, tailored to particular symptom and comorbidity profiles in OSA.

\section{EHR-derived evidence supports influences of SNPs in GABBR1 and LEPR on OSA diagnosis and variables from sleep studies}

Arguably, the GABBR1 variant (rs29230) had the most consistent evidence for an association with OSA-related traits in European Americans in our sample, including a significant association with increased risk for OSA in meta-analysis $\left(\mathrm{OR}=1.06, p=8.44 \times 10^{-3}\right)$ and evidence of an association with increased wake after sleep onset $\left(p=1.73 \times 10^{-3}\right)$. The association between this SNP and OSA was reported in a candidate gene study conducted in 174 individuals from Turkey, where OSA was defined as apnea hypopnea index (AHI) $\geq 5$ and controls were confirmed using medical histories [28]; the SNP was associated primarily among males [28]. A subsequent study of 266 individuals from China confirmed the association of this SNP with OSA [29]. GABBR1 encodes a receptor for the main inhibitory neurotransmitter in humans, gamma-aminobutyric acid (GABA). GABBR1 variation may influence risk for OSA by affecting activity of the encoded receptor, which is expressed in 


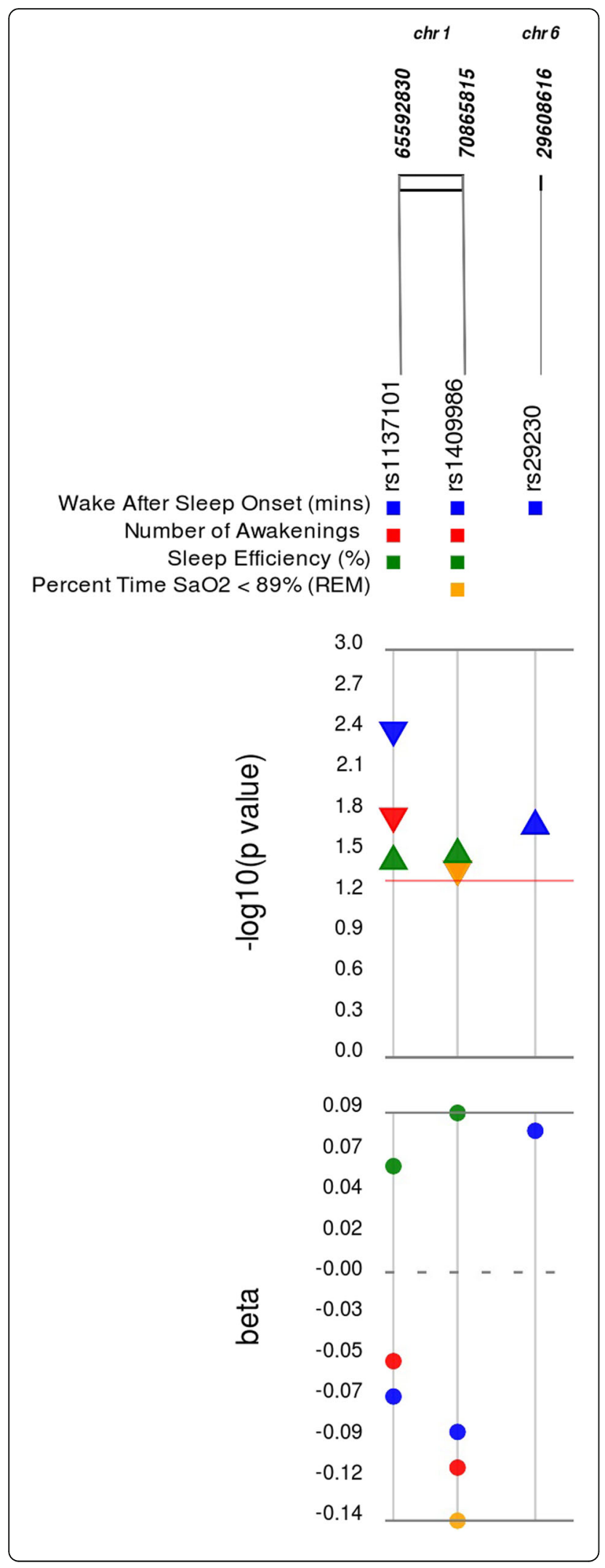

Fig. 2 Associations between Literature-derived Candidate SNPs and Sleep Study Report Variables. Plotted are the estimates of the additive effects (beta) of the minor allele at each candidate SNP reported to be associated with OSA in populations of European ancestry on variables obtained from sleep study reports available in the Geisinger dataset only, along with the corresponding $-\log _{10} p$ value. For each SNP, the rsID, chromosome and hg38 base pair location are provided. Colors of betas and $p$-values reflect those obtained for the corresponding sleep study report variable listed above. For $p$-values, up arrows denote increases in variable measurements and down arrows denote decreases. Red line denotes unadjusted significance threshold $\left(p<5.0 \times 10^{-2}\right)$

hypoglossal motor neurons innervating the tongue, resulting in altered inhibition of tongue movement [28, 29]. The predicted consequences of this SNP vary across different in silico algorithms, with some reporting the variant to be damaging and others benign (Ensembl Variant Effect Predictor, https://uswest.ensembl.org); thus, additional functional characterization of this SNP is necessary to elucidate a mechanistic role in OSA risk.

There was also a missense variant in $L E P R$ (rs1137101) that had substantial evidence for a relationship with OSA in European Americans at Geisinger, including decreased risk for EHR-defined OSA (OR $=0.95$, $\left.p=3.78 \times 10^{-2}\right)$ and associations with increased sleep efficiency $\left(p=2.28 \times 10^{-3}\right)$, reduced wake after sleep onset $\left(p=2.64 \times 10^{-4}\right)$, fewer awakenings $\left(p=9.40 \times 10^{-3}\right)$ and shorter respiratory event duration $\left(p=3.01 \times 10^{-2}\right)$. Despite this evidence, the variant was not associated in the VUMC-EA dataset or in joint meta-analysis. Previous meta-analysis of seven candidate gene studies observed an association between rs1137101 and decreased risk for OSA specifically in Europeans, but not in Asians [30]. $L E P R$ encodes a receptor for the adipocyte-specific leptin hormone and there is evidence of a relationship between leptin levels and OSA [30]. Higher levels of leptin have been observed in OSA patients compared to controls [31], and the AHI has been shown to be an independent predictor of the evening/morning leptin ratio, suggesting that OSA might affect leptin diurnal rhythms [32]. Given the relationship between OSA and obesity, it is notable that this SNP is not associated with risk for obesity [3335], suggesting $L E P R$ genetic variation has effects on OSA that are independent of this established pathway.

Among variants associated with quantitative EHR phenotypes, one SNP demonstrated inconsistent evidence when compared to previous literature. The A allele at the rs1409986 SNP, located in a non-coding transcript of the PTGER3 gene, was previously associated with increased OSA risk-defined based on AHI $\geq 15$-in an extensive candidate gene study of 2904 individuals of European ancestry [36]. In our analyses, the rs1409986 SNP was not associated with OSA diagnosis. Additionally, the previous study failed to replicate the association 
Table 3 Associations between Obstructive Sleep Apnea Candidate SNPs and Other EHR-derived Clinical Traits

\begin{tabular}{|c|c|c|c|c|c|c|c|c|c|c|}
\hline \multirow[t]{2}{*}{ SNP } & \multirow[t]{2}{*}{ Chr } & \multirow{2}{*}{$\begin{array}{l}\text { Closest } \\
\text { Gene }\end{array}$} & \multirow[t]{2}{*}{ EHR-Derived Trait } & \multirow{2}{*}{$\begin{array}{l}\text { Effect } \\
\text { Allele }\end{array}$} & \multicolumn{2}{|c|}{ Geisinger (EA) } & \multirow{2}{*}{$\begin{array}{l}\text { VUMC (EA) } \\
\beta \text { (SE) }\end{array}$} & \multicolumn{3}{|l|}{ VUMC (AA) } \\
\hline & & & & & $\beta(\mathrm{SE})$ & q-value & & q-value & $\beta$ (SE) & q-value \\
\hline \multirow[t]{2}{*}{ rs2187668 } & $6 p 21.32$ & HLA & Celiac Disease & $T^{a}$ & $1.62(0.12)$ & $5.80 \times 10^{-38}$ & $1.15(0.34)$ & $2.83 \times 10^{-3}$ & \multicolumn{2}{|c|}{$\begin{array}{l}\text { Non-varying phenotype/ } \\
\text { genotype }\end{array}$} \\
\hline & & & Hypothyroidism NOS & & $0.15(0.03)$ & $7.67 \times 10^{-4}$ & $0.22(0.08)$ & $1.13 \times 10^{-2}$ & $0.44(0.24)$ & $3.10 \times 10^{-1}$ \\
\hline \multirow[t]{3}{*}{ rs429358 } & $19 q 13.32$ & $A P O E$ & Hyperlipidemia & $C$ & $0.26(0.03)$ & $7.57 \times 10^{-20}$ & $0.16(0.04)$ & $3.86 \times 10^{-4}$ & $0.36(0.11)$ & $4.69 \times 10^{-3}$ \\
\hline & & & HDL-Cholesterol ${ }^{\mathrm{b}}$ & & $-1.51(0.15)$ & $8.80 \times 10^{-19}$ & $-5.95(1.35)$ & $1.82 \times 10^{-4}$ & $-2.21(2.62)$ & $5.59 \times 10^{-1}$ \\
\hline & & & Mixed hyperlipidemia & & $0.17(0.04)$ & $3.97 \times 10^{-3}$ & $0.20(0.05)$ & $1.93 \times 10^{-4}$ & $0.39(0.11)$ & $4.69 \times 10^{-3}$ \\
\hline rs7412 & $19 q 13.32$ & & Hyperlipidemia & $\mathrm{T}$ & $-0.46(0.03)$ & $9.88 \times 10^{-46}$ & $-0.21(0.06)$ & $1.01 \times 10^{-3}$ & $-0.22(0.14)$ & $3.42 \times 10^{-1}$ \\
\hline
\end{tabular}

Shown are phenome-wide association test results where presence of the clinical code for the respective disorder on $\geq 3$ different dates in the EHR was associated with the OSA candidate SNP at FDR-corrected $\mathrm{q}<5.0 \times 10^{-2}$ in the Geisinger dataset, and replicated in the Vanderbilt University Medical Center European American (EA) dataset. ${ }^{a}$ Literature-reported effect allele located on the negative strand. ${ }^{b}$ Indicates an association with the median laboratory value for this measure in serum or plasma. Indicates test could not be conducted due to non-varying phenotype or genotype in either cases or controls. Abbreviations: SNP Single nucleotide polymorphism, Chr Chromosome, EHR Electronic health record, SE Standard error, AA African American, NOS Not otherwise specified, HDL Highdensity lipoprotein. All SNPs evaluated in EHR-based datasets are mapped to the positive strand. See Table S7 for more details

between rs1409986 and OSA risk in analyses of independent datasets, indicating that this association may not generalize across distinct analysis datasets [36]. Furthermore, differences in definitions of PSG-based OSA diagnosis could explain why an association with EHRdefined OSA diagnosis did not validate. The study reporting the original association between rs1409986 and OSA defined case status as an AHI $\geq 15$ [36], while to qualify for a diagnosis of OSA based on EHR-derived clinical codes it was necessary to have clinical codes used on more than one date in the EHR. Clinical codes for OSA in the EHR are likely used once in order to obtain a diagnostic sleep study, and a second or third code would then be used following a PSG with an AHI meeting the American Academy of Sleep Medicine criteria for OSA (i.e., AHI > 5).

While not associated with OSA diagnosis, we did observe associations between the rs1409986 SNP and less time spent at low oxygen saturation, decreased number of awakenings and increased sleep efficiency. The original study reporting this association evaluated log-transformed AHI, but not other measures from the PSG, and did not observe a significant relationship that survived multiple testing correction (uncorrected $\mathrm{p}=2.0 \times 10^{-2}$ ) between genotypes at the rs1409986 SNP and log-transformed AHI [36]. Notably, PSG-derived measures of sleep quality (e.g., number of awakenings, sleep efficiency, wake after sleep onset) are not specific to OSA and are more often used to define insomnia. As such, it is difficult to know whether our results reflect evidence that this SNP is associated with less severe symptoms of OSA, or insomnia. It is also notable that both the GABBR1 and the $L E P R$ variant were associated with measures of sleep quality in addition to OSA-related traits. Interestingly, our group has observed a distinct subgroup of individuals with OSA who have insomnia- related symptoms [37]. These associations may reflect genetically-distinct phenotypic subgroups of OSA with comorbid insomnia.

\section{Associations of OSA candidate SNPs may reflect underlying comorbidities}

Beyond OSA diagnosis and severity, we evaluated associations between OSA candidate SNPs and other EHR-based phenotypes, which can provide evidence of pleiotropic genetic effects or indicate that original associations were driven by genetic effects on OSArelated comorbidities. Several SNPs previously associated with OSA showed significant associations with EHR phenotypes. Of particular interest were SNPs tagging the $A P O E-\varepsilon 2 \quad($ rs7412) and $A P O E-\varepsilon 4$ (rs429358) alleles, as well as a variant in the $H L A$ gene (rs2187668). Specifically, the APOE-E2 tagging SNP previously shown to be protective for OSA was not associated with EHR-defined OSA diagnosis. Notably, rs7412 was instead associated with a lower risk of hyperlipidemia in both Geisinger and VUMC-EA. It is feasible that prior associations with decreased risk of OSA may be driven by this underlying association, as patients with OSA are at higher risk for metabolic syndrome [38]. Similarly, while the APOEE4 tagging SNP (rs429358) previously shown to increase OSA risk was not associated with EHR-defined OSA in our sample, this SNP was associated with increased risk for hyperlipidemia and lower levels of HDL cholesterol. These associations are supported by prior literature [26], which observed that $A P O E-\varepsilon 2$ carriers had smaller LDL with normal HDL, and $A P O E-E 4$ carriers had smaller HDL. Once again, it is feasible that the original association was driven by the effect of this $A P O E$ variant on underlying cardiometabolic disease, rather than a true association with OSA itself. 
Finally, the HLA SNP previously implicated in OSA risk was not associated with OSA-related phenotypes in our sample, but showed effects on increased risk for autoimmune thyroid diseases and celiac disease. Hypothyroidism is considered a risk factor for OSA, with as many as $35 \%$ of individuals having comorbid or eventual OSA [39]. Potential mechanisms include deposition of mucoprotein in the upper airway, reduced neural output to airway musculature, abnormal ventilatory control, and a dual relationship with obesity [40]. Similarly, a connection between OSA and celiac disease has been proposed [41]. Specifically, symptoms of celiac disease (e.g. gastroesophageal reflux disease) may contribute to disturbed sleep; however, evidence suggests that sleep disorders in individuals with celiac disease are independent of gastrointestinal issues [41]. Furthermore, celiac disease and OSA share common features of lymphatic hyperplasia and local inflammation, and studies conducted in children report an increased prevalence of OSA in patients with celiac disease [42]. There was no mention in the original study reporting an association between this SNP and OSA of excluding individuals with thyroid disorders or celiac disease [43]. As such, it is possible that a proportion of OSA cases in the original study had underlying comorbidities that were contributing to expression of OSA, which explains the observed association with OSA itself (e.g., mediated pleiotropy). These individuals may represent a distinct OSA patient subgroup not strongly represented among patients in our large EHR-based sample.

\section{Additional reasons for lack of validation}

A majority of the candidate SNPs we examined did not validate for an association with OSA diagnosis or severity, or associate with other phenotypes in the EHR. As discussed above, this lack of replication may result from limitations in our EHR-based OSA phenotypes or could reflect underlying associations with OSA comorbidities, rather than the disease itself. Ultimately, lack of replication is a common problem in genetic analyses, as initial discovery analyses are more likely to overestimate the true associations (e.g., "Winner's Curse") [44]. This can be particularly problematic in candidate gene studies, which tend to rely on smaller sample sizes that are more prone to spurious associations. As described by Varvarigou et al [45], small and underpowered genetic studies, typically lacking replication, are particularly problematic for OSA genetic research. As a result of these issues, it is feasible that a majority of the identified candidate SNPs are false positive associations. Beyond this, the effects of these variants may not generalize to all ancestral populations, as approximately one-third of the SNPs tested in our dataset were identified in populations with different ancestral backgrounds (e.g., Latin American, East Asian).
The established heterogeneity in the causes of OSA, including obesity-related pathways [46], specific craniofacial morphologies [47], and physiological mechanisms [48], may also explain the difficulties in replicating associations in large-scale clinical populations like those studies here. Combining individuals with various disease etiologies with potentially distinct genetic influences can obscure underlying associations. Towards this end, careful characterization of OSA cases with respect to disease subtypes and consequences may be required to identify reproducible genetic effects.

\section{Strengths and limitations}

Strengths of this study include the large sample size studied across the two health systems, the detailed information leveraged from electronic health records, and the application of robust association methods to comprehensively evaluate the role of OSA candidate variants.

There are also a number of limitations. A limitation of testing associations between genomic variants and EHRderived OSA diagnosis is the possibility of undiagnosed OSA among non-cases. Prior studies suggest that a high percentage of individuals referred for sleep studies have undiagnosed OSA [49]; however, these studies are potentially biased given the elevated risk among those referred for testing. In our recent validation study [19], $\sim 70 \%$ of non-cases had a predicted OSA probability below $20 \%$ and fewer than $3 \%$ had a predicted probability above $70 \%$, based on the symptomless multi-variable apnea prediction score [50]. Thus, most individuals defined in this study as non-cases are likely true controls. Ultimately, bias introduced by including individuals with undiagnosed OSA in the control group should make it more difficult to identify associations and will not affect the validated associations that we found; however, this could explain negative results. Notably, we complement tests for associations between SNPs and OSA diagnosis with tests between SNPs and OSA severity measures. This should further account for bias related to inaccurate control definitions. Future research using EHR datasets would benefit from studies focused on accurate identification of undiagnosed OSA. Towards this end, obtaining raw sleep study files on large number of patients, both to confirm OSA diagnoses and expand the characterization of quantitative phenotypes, would be beneficial. In addition, while our sample was larger than nearly all of the original studies in which candidate SNPs were identified, the relatively small sample size for specific analyses, particularly among African Americans at VUMC and in quantitative analyses only available in the Geisinger sample, may have limited statistical power for detecting associations. This may be particularly impactful given the expectation that associations in our sample will be smaller than those observed in the original 
discovery analyses [44]. Finally, to maximize our ability to validate previous associations reported in ancestral populations that are comparable to our study populations, we defined statistical significance for validation of many previously reported associations based on a $p<$ $5.0 \times 10^{-2}$ in analyses of EHR-based OSA diagnosis, which could increase the likelihood of a false positive result. Secondary analyses of quantitative OSA phenotypes and PheWAS analyses were adjusted for multiple comparisons. Ultimately, even at this liberal threshold, a majority of SNPs did not show associations with our endpoints of interest. As such, future work aimed at conducting larger, well-powered genome-wide analyses to assess OSA genetic risk and genetic effects on OSA severity across populations with diverse ancestral backgrounds are warranted.

\section{Conclusions}

This manuscript leverages the breadth of available clinical information in the EHR to comprehensively evaluate the relationships between candidate genetic variants related to OSA and both OSA and non-OSA phenotypes. We validated associations between a small portion of the previously reported OSA candidate SNPs and OSA diagnosis, including variation related to the genes GABBR1, $L E P R$ and $M M P-9$, and observed several associations with OSA-related traits from sleep study reports. However, a majority of the candidate SNPs did not show any OSA association in our sample, suggesting these may be false positive results. SNPs related to APOE and HLA showed stronger associations with non-OSA related phenotypes of hyperlipidemia, cholesterol, thyroid disease and/or celiac disease. Relationships between these conditions and risk for OSA may explain the original associations, or suggest pleiotropic genetic effects. Ultimately, our results reflect the remarkable resources that EHRderived datasets offer for characterizing the genetic and phenotypic heterogeneity of OSA.

\section{Supplementary information}

Supplementary information accompanies this paper at https://doi.org/10. 1186/s12920-020-00755-4.

\footnotetext{
Additional file 1 Figure S1. Inclusion Criteria for Reviewed Studies and Selection of Candidate Variants. Overview of the systematic literature review procedures, and the process for selecting candidate single nucleotide polymorphisms (SNPs) to test for associations with obstructive sleep apnea (OSA) diagnosis, sleep study report variables and phenome-wide clinical traits. Abbreviations: in/del = insertion/deletion variant, $L D=$ linkage disequilibrium, $M A F=$ minor allele frequency.

Additional file 2. Table S1. Clinical Codes Used to Define Sleep Apnea.

Additional file 3. Table S2. International Classification of Diseases, Ninth Edition Codes and Corresponding PheCodes Tested for Associations with OSA Candidate Variants.
}

Additional file 4. Table S3. Logical Observation Identifier Names and Codes Mapped Median Laboratory Values Tested for Associations with OSA Candidate Variants.

Additional file 5. Table S4. Association Tests for Effects of Candidate SNPs, obtained via Literature Reviews, on Electronic Health Recordderived Obstructive Sleep Apnea (OSA).

Additional file 6. Table S5. Results of Interaction Tests for the Effects of Age, Sex and Body Mass Index on Associations between Candidate Variants and Obstructive Sleep Apnoea Diagnosis.

Additional file 7. Table S6. Results from Tests for Associations between Obstructive Sleep Apnea Candidate SNPs and Variables Obtained from Sleep Reports at Geisinger.

Additional file 8. Table S7. Significant Results from Phenome-wide Studies Testing the Associations between Obstructive Sleep Apnoea Candidate SNPs and Other Clinical Codes and Lab Values.

\section{Abbreviations}

OSA: Obstructive Sleep Apnea; EHR: Electronic Health Record; SNP: Single Nucleotide Polymorphism; VUMC: Vanderbilt University Medical Center; PSG: Polysomnography; AHI: Apnea/Hypopnea Index; PheWAS: Phenomewide association studies; EA: European Americans; AA: African Americans; QC: Quality Control; ICD: International Classification of Diseases; LOINC: Logical Observation Identifier Names and Codes; BMI: Body Mass Index

\section{Acknowledgements}

We would like to acknowledge the resources graciously provided by the Geisinger MyCode project and the Biorepository of Vanderbilt University (BioVU). Vanderbilt University's BioVU projects are supported by numerous sources: institutional funding, private agencies, and federal grants.

\section{Authors' contributions}

AIP, JDR, \& SAP conceived of the presented idea. OJV, CRB, DRM, BTK \& SAP designed the analysis plan with critical feedback from JDR, KB, BAM \& AIP. OJV, DRM \& BTK conducted the literature review. OJV, CRB \& NJ conducted all analyses. OJV drafted the initial manuscript. All authors discussed contributed to the final manuscript. The authors read and approved the final manuscript.

\section{Funding}

This work was directly supported by the following grants: R01HL134015, K01LM012870, P01HL094307, AASM Foundation 194-SR-18, Dept of Health 4100070267 . No funding agencies contributed to the study design, data collection, data analysis, interpretation or manuscript writing.

\section{Availability of data and materials}

The data that support the findings of this study are available from Geisinger and VUMC/BioVU but restrictions apply to the availability of these data, which were used under license for the current study, and so are not publicly available. Data are however available from the authors upon reasonable request and with permission of Geisinger or VUMC/BioVU.

\section{Ethics approval and consent to participate}

MyCode participants are consented under an IRB-approved protocol that allows research across a broad range of clinical conditions and permits the sharing of data consistent with the NIH data sharing policy. The Medical Center Ethics Committee was consulted during the planning phase of the BioVU biorepository and continues to provide oversight. The Vanderbilt IRB have on-going oversight; all patients had the right to receive information about this project through a comprehensive education plan, as well as the right to refuse to participate by opting out of the program.

Consent for publication

Not applicable.

Competing interests

The authors have no conflicts of interest to disclose. 


\section{Author details}

'Division of Sleep Medicine/Department of Medicine, Perelman School of Medicine at the University of Pennsylvania, 125 S. 31st St, Office 2123, Philadelphia, PA 19104, USA. ${ }^{2}$ Sleep Disorders Division/Department of Neurology, Vanderbilt University Medical Center, Nashville, TN 37232, USA. ${ }^{3}$ Department of Psychiatry \& Behavioral Sciences, University of Kansas Medical Center, Mail-Stop 4015, 3901 Rainbow Blvd., Kansas City, KS 66160, USA. ${ }^{4}$ Geisinger Research, Rockville, MD 20852, USA. ${ }^{5}$ Department of Biomedical Science, Charles E. Schmidt College of Medicine, Florida Atlantic University, Boca Raton, FL 33431, USA.

\section{Received: 12 December 2019 Accepted: 13 July 2020} Published online: 25 July 2020

\section{References}

1. Benjafield AV, Ayas NT, Eastwood PR, Heinzer R, MSM I, Morrell MJ, et al. Estimation of the global prevalence and burden of obstructive sleep apnoea: a literature-based analysis. Lancet Respir Med. 2019;7(8):687-98.

2. Pack Al, Gislason T. Obstructive sleep apnea and cardiovascular disease: a perspective and future directions. Prog Cardiovasc Dis. 2009;51(5):434-51 EPUB 2009/03/03.

3. Gozal D, Ham SA, Mokhlesi B. Sleep apnea and cancer: analysis of a nationwide population sample. SLEEP. 2016;39(8):1493-500 EPUB 2016/05/ 12.

4. Verstraeten E. Neurocognitive effects of obstructive sleep apnea syndrome Curr Neurol Neurosci Rep. 2007;7(2):161-6 EPUB 2007/02/28.

5. Yaffe K, Laffan AM, Harrison SL, Redline S, Spira AP, Ensrud KE, et al. Sleepdisordered breathing, hypoxia, and risk of mild cognitive impairment and dementia in older women. JAMA. 2011;306(6):613-9 EPUB 2011/08/11.

6. Mukherjee $S$, Saxena R, Palmer $L$. The genetics of obstructive sleep APNOEA. Respirology. 2018;23(1):18-27 EPUB 2017/11/08.

7. Schwab RJ, Pasirstein M, Kaplan L, Pierson R, Mackley A, Hachadoorian R, et al. Family aggregation of upper airway soft tissue structures in normal subjects and patients with sleep apnea. Am J Respir Crit Care Med. 2006; 173(4):453-63.

8. Chi L, Comyn FL, Keenan BT, Cater J, Maislin G, Pack Al, et al. Heritability of craniofacial structures in normal subjects and patients with sleep apnea. Sleep. 2014;37(10):1689-98 EPUB 2014/09/10.

9. Xia Q, Grant SF. The genetics of human obesity. Ann N Y Acad Sci. 2013; 1281:178-90 EPUB 2013/01/31.

10. Afsharpaiman S, Saburi A, Waters KA. Respiratory difficulties and breathing disorders in Achondroplasia. Paediatr Respir Rev. 2013;14(4):250-5 EPUB 2013/03/26

11. Trois MS, Capone GT, Lutz JA, Melendres MC, Schwartz AR, Collop NA, et al. Obstructive sleep apnea in adults with down syndrome. J Clin Sleep Med. 2009:5(4):317-23 EPUB 2009/12/09.

12. Mo L, He Q, Wang $Y$, Dong $B$, He J. High prevalence of obstructive sleep apnea in MARFAN'S syndrome. Chin Med J (Engl). 2014;127(17):3150-5 EPUB 2014/09/06.

13. Sedky K, Bennett DS, Pumariega A. Prader willi syndrome and obstructive sleep apnea: co-occurrence in the pediatric population. J Clin Sleep Med. 2014;10(4):403-9 EPUB 2014/04/16.

14. Pendergrass SA, Brown-Gentry K, Dudek SM, Torstenson ES, Ambite JL, Avery $\mathrm{CL}$, et al. The use of PHENOME-wide association studies (PHEWAS) for exploration of novel genotype-phenotype relationships and PLEIOTROPY discovery. Genet Epidemiol. 2011;35(5):410-22 EPUB 2011/05/20.

15. Auton A, Brooks LD, Durbin RM, Garrison EP, Kang HM, Korbel JO, et al. A global reference for human genetic variation. Nature. 2015;526(7571):68-74 EPUB 2015/10/04.

16. Carey DJ, Fetterolf SN, Davis FD, Faucett WA, Kirchner HL, Mirshahi U, et al. The GEISINGER MYCODE community health initiative: an electronic health record-linked biobank for precision MEDICINE research. Genet Med. 2016; 18(9):906-13 EPUB 2016/02/13.

17. Crawford DC, Goodloe R, Farber-Eger E, Boston J, Pendergrass SA, Haines JL, et al. Leveraging epidemiologic and clinical collections for genomic studies of complex traits. Hum Hered. 2015;79(3-4):137-46 EPUB 2015/07/24.

18. Mccarthy S, Das S, Kretzschmar W, Delaneau O, Wood AR, Teumer A, et al. A reference panel of 64,976 haplotypes for genotype imputation. Nat Genet. 2016;48(10):1279-83 EPUB 2016/08/23.

19. Keenan BT, Kirchner HL, Veatch OJ, Borthwick KM, Davenport VA, Feemster $\mathrm{JC}$, et al. Multi-site validation of a simple electronic health record algorithm for identifying diagnosed obstructive sleep apnea. J Clin SLEEP MED. 2020; 16(2):175-183. https://doi.org/10.5664/jcsm.8160. Epub 2020 Jan 13.

20. Johns MW. A new method for measuring daytime sleepiness: the Epworth sleepiness scale. Sleep. 1991;14(6):540-5 EPUB 1991/12/01.

21. Goodloe R, Farber-Eger E, Boston J, Crawford DC, Bush WS. Reducing clinical noise for body mass index measures due to unit and transcription errors in the electronic health record. AMIA Joint Summits Transl Sci Proc AMIA Joint Summits Transl Sci. 2017;2017:102-11 EPUB 2017/08/18.

22. Cronin RM, Field JR, Bradford Y, Shaffer CM, Carroll RJ, Mosley JD, et al. Phenome-wide association studies demonstrating pleiotropy of genetic variants within FTO with and without adjustment for body mass index. Front Genet. 2014:5:250 EPUB 2014/09/02.

23. Willer CJ, Li Y, Abecasis GR. Metal: fast and efficient meta-analysis of genomewide association scans. BIOINFORMATICS. 2010;26(17):2190-1 EPUB 2010/07/10.

24. Bauer CR, Lavage D, Snyder J, Leader J, Mahoney JM, Pendergrass SA. Opening the door to the large scale use of clinical lab measures for association testing: exploring different methods for defining phenotypes. Pacific Symp Biocomput Pacific Symp Biocomput. 2017;22:356-67 EPUB 2016/11/30

25. Wei WQ, Bastarache LA, Carroll RJ, Marlo JE, Osterman TJ, Gamazon ER, et al. Evaluating phecodes, clinical classification software, and ICD-9-cm codes for phenome-wide association studies in the electronic health record. PLoS One. 2017;12(7):E0175508 EPUB 2017/07/08.

26. Marais AD. Apolipoprotein e in lipoprotein metabolism, health and cardiovascular disease. Pathology. 2019;51(2):165-76 EPUB 2019/01/02

27. Babron MC, Nilsson S, Adamovic S, Naluai AT, Wahlstrom J, Ascher $\mathrm{H}$, et al. Meta and pooled analysis of EUROPEAN coeliac disease data. Eur J Human Genet. 2003;11(11):828-34 EPUB 2003/10/23.

28. Bayazit YA, Yilmaz M, Kokturk O, Erdal ME, Ciftci T, Gokdogan T, et al. Association of GABA(b)R1 receptor gene polymorphism with obstructive sleep apnea syndrome. ORL J Otorhinolaryngol Relat Spec. 2007;69(3):190-7 EPUB 2007/02/01.

29. Li Z, Tang T, Du J, Wu W, Zhou X, Qin G. Association between single nucleotide polymorphisms in gamma-AMINOBUTYRIC acid b receptor, insulin receptor SUBSTRATE-1, and HYPOCRETIN neuropeptide precursor genes and susceptibility to obstructive SLEEP apnea hypopnea syndrome in a CHINESE HAN population. Med Principles Pract. 2016;25(6):517-24 EPUB 2016/08/11

30. Lv D, Tan L, Wu Y, Cao C, Deng Z. Leptin and leptin receptor gene polymorphisms in obstructive sleep apnea: a huge review and METAanalysis. Sleep Breathing = Schlaf \& Atmung. 2015;19(3):1073-8 EPUB 2015/ $01 / 21$.

31. Ulukavak Ciftci T, Kokturk O, Bukan N, Bilgihan A. Leptin and ghrelin levels in patients with obstructive sleep apnea syndrome. Respiration; Int Rev Thoracic Dis. 2005;72(4):395-401 EPUB 2005/08/10.

32. Patel SR, Palmer LJ, Larkin EK, Jenny NS, White DP, Redline S. Relationship between obstructive sleep apnea and diurnal leptin rhythms. SLEEP. 2004; 27(2):235-9.

33. Olza J, Ruperez Al, Gil-Campos M, Leis R, Canete R, Tojo R, et al. Leptin receptor gene variant RS11804091 is associated with BMI and insulin resistance in SPAN ISH female obese children: a CASE-control study. Int J Mol Sci. 2017;18(8):1690. https://doi.org/10.3390/ijms18081690. EPUB 2017/08/05.

34. Rojano-Rodriguez ME, Beristain-Hernandez JL, Zavaleta-Villa B, Maravilla P, Romero-Valdovinos M, Olivo-Diaz A. Leptin receptor gene polymorphisms and morbid obesity in MEXICAN patients. Hereditas. 2016;153:2 EPUB 2017/01/18.

35. Chavarria-Avila E, Vazquez-Del MERCADOM, Gomez-Banuelos E, RuizQuezada SL, Castro-Albarran J, Sanchez-Lopez L, et al. The impact of LEP G2548A and LEPR GLN223ARG polymorphisms on adiposity, leptin, and leptin-receptor serum levels in a mexican mestizo population. Biomed Res Int. 2015;2015:539408 EPUB 2015/06/13.

36. Patel SR, Goodloe R, De G, Kowgier M, Weng J, Buxbaum SG, et al. Association of genetic loci with sleep apnea in european americans and african-americans: the candidate gene association resource (care). PLoS One. 2012;7(11):E48836 EPUB 2012/11/17.

37. Keenan BT, Kim J, Singh B, Bittencourt L, Chen NH, Cistulli PA, et al. Recognizable clinical subtypes of obstructive sleep apnea across international sleep centers: a cluster analysis. sleep. 2018;41(3) EPUB 2018/01/10.

38. Qian Y, Xu H, Wang Y, Yi H, Guan J, Yin S. Obstructive sleep apnea predicts risk of metabolic syndrome independently of obesity: a meta-analysis. Arch Med Sci. 2016;12(5):1077-87 EPUB 2016/10/04. 
39. Bozkurt NC, Karbek B, Cakal E, Firat H, Ozbek M, Delibasi T. The association between severity of obstructive sleep apnea and prevalence of hashimoto's thyroiditis. Endocr J. 2012;59(11):981-8 EPUB 2012/07/13.

40. Devdhar M, Ousman YH, Burman KD. Hypothyroidism. Endocrinol Metab Clin North Am. 2007;36(3):595-615 V. EPUB 2007/08/04.

41. Zingone F, Siniscalchi M, Capone P, Tortora R, Andreozzi P, Capone E, et al, The quality of sleep in patients with coeliac disease. Aliment Pharmacol Ther. 2010;32(8):1031-6 EPUB 2010/10/13.

42. Yerushalmy-Feler A, Tauman R, Derowe A, Averbuch E, Ben-Tov A, Weintraub $Y$, et al. Gluten-free diet may improve obstructive sleep apnearelated symptoms in children with celiac disease. BMC Pediatr. 2018;18(1):35 EPUB 2018/02/09.

43. Silva L, Lopes J, Ramalheira J, Cunha D, Carvalho C, Bettencourt A, et al. Obstructive sleep apnoea syndrome and HLA in the north of Portugal. Rev Neurol. 2015:61(7):301-7 EPUB 2015/09/29.

44. Ioannidis JP. Why MOST discovered true associations are inflated. Epidemiology. 2008;19(5):640-8 EPUB 2008/07/18.

45. Varvarigou V, Dahabreh IJ, Malhotra A, Kales SN. A review of genetic association studies of obstructive sleep apnea: FIELD synopsis and METAanalysis. Sleep. 2011;34(11):1461-8 EPUB 2011/11/02.

46. Patel SR. Shared genetic risk factors for obstructive sleep apnea and obesity. J Appl Physiol. 2005;99(4):1600-6 EPUB 2005/09/15.

47. Schwab RJ, Pasirstein M, Pierson R, Mackley A, Hachadoorian R, Arens R, et al. Identification of upper airway anatomic risk factors for obstructive sleep apnea with volumetric magnetic resonance imaging. Am J Respir Crit Care Med. 2003:168(5):522-30 EPUB 2003/05/15

48. Eckert DJ, White DP, Jordan AS, Malhotra A, Wellman A. Defining phenotypic causes of obstructive sleep apnea. Identification of novel therapeutic targets. Am J Respir Crit Care Med. 2013;188(8):996-1004 EPUB 2013/06/01.

49. Laratta CR, Tsai WH, Wick J, Pendharkar SR, Johannson KA, Ronksley PE. Validity of administrative data for identification of obstructive sleep apnea. J Sleep Res. 2017:26(2):132-8 EPUB 2016/10/21.

50. Lyons MM, Keenan BT, Li J, Khan T, Elkassabany N, Walsh CM, et al. Symptomless multi-variable apnea prediction index assesses obstructive sleep apnea risk and adverse outcomes in elective surgery. Sleep. 2017;40(3) EPUB 2017/04/02.

\section{Publisher's Note}

Springer Nature remains neutral with regard to jurisdictional claims in published maps and institutional affiliations.

Ready to submit your research? Choose BMC and benefit from:

- fast, convenient online submission

- thorough peer review by experienced researchers in your field

- rapid publication on acceptance

- support for research data, including large and complex data types

- gold Open Access which fosters wider collaboration and increased citations

- maximum visibility for your research: over $100 \mathrm{M}$ website views per year

At $\mathrm{BMC}$, research is always in progress.

Learn more biomedcentral.com/submissions 